\title{
Histopathological Analyses of Proliferative Potentials of Intracranial Meningiomas Using Bromodeoxyuridine and MIB-1 Immunohistochemistry
}

\author{
Akira Matsuno ${ }^{1}$, Hiroshi Nakaguchi ${ }^{1}$, Tadashi Nagashima ${ }^{1}$, Takamitsu Fujimaki ${ }^{2}$ and \\ R. Yoshiyuki Osamura ${ }^{3}$ \\ ${ }^{1}$ Department of Neurosurgery, Teikyo University Ichihara Hospital, 3426-3 Anesaki, Ichihara City, Chiba 299-0111, Japan, \\ ${ }^{2}$ Department of Neurosurgery, Teikyo University Itabashi Hospital, 2-11-1 Kaga, Itabashi-ku, Tokyo 173-0003, Japan and \\ ${ }^{3}$ Department of Pathology, Tokai University School of Medicine, Boseidai, Isehara City, Kanagawa 259-1100, Japan
}

Received November 4, 2004; accepted January 31, 2005

The proliferative potentials of intracranial meningiomas, as represented by bromodeoxyuridine (BrdU) labeling index (LI) and MIB-1 staining index (SI), were examined, and their correlation with recurrence and regrowth of meningiomas was reviewed. The mean BrdU LI of recurrent meningiomas was reported to be significantly higher than that of nonrecurrent meningiomas. BrdU LI can predict the time to re-operation. Many studies have been conducted to examine the relationship between MIB-1 SI and clinical outcome of meningiomas, and most of them showed a significant relationship. Time interval to next recurrence in recurrent meningiomas is associated with MIB-1 SI, and several investigators have shown a cut-off point for MIB-1 SI that can predict future recurrence. BrdU LI, MIB-1 SI, and tumor doubling time of intracranial meningiomas have a mutual relationship. While many other biological or genetic variables can affect the proliferation of tumors, nevertheless BrdU LI and MIB-1 SI are closely correlated with clinical recurrence or regrowth of meningiomas.

Key words: meningioma, BrdU, MIB-1, proliferative potential, recurrence

\section{Introduction}

Meningiomas are common, usually benign tumors of the meninges arising from the arachnoid cells. They comprise a heterogeneous group of tumors that is categorized into 15 subtypes [27]. Several investigators have studied the clinical and biological features of recurrent and nonrecurrent meningiomas $[3,8,10,11,14,20,21,31,35,36$, 52]. In recent years, meningiomas have been analyzed by histopathological examination focused on their proliferative potentials. Representative studies on proliferative potentials include bromodeoxyuridine (BrdU) labeling index (LI) $[16,22,51]$, proliferative cell nuclear antigen (PCNA) [24],

Correspondence to: Akira Matsuno, M.D., Ph.D., Department of Neurosurgery, Teikyo University Ichihara Hospital, 3426-3 Anesaki, Ichihara City, Chiba 299-0111, Japan.

E-mail: akirakun@med.teikyo-u.ac.jp argyrophilic nucleolar organizer region (AgNOR) count [4, 9, 45], and Ki-67 or MIB-1 staining index (SI) [1, 2, 15, 18, $22,23,26,28-30,32,33,37,40,42,43,46-49,54,55]$. In this paper, proliferative potentials of intracranial meningiomas, especially represented by BrdU LI and MIB-1 SI, were examined, and their correlation with recurrence and tumor growth was reviewed.

\section{BrdU LI and Recurrence}

BrdU can detect $\mathrm{S}$ phase cells alone $[16,51,53]$, and histopathological studies using BrdU LI require intravenous injection of BrdU prior to surgery, or incubation of cultured tumor cells with BrdU. The method of BrdU immunohistochemical stainings was described in several reports [16, $17,22,41,51]$.

Hoshino et al. analyzed BrdU LIs of 20 intracranial meningiomas [16]. In their report, the average LIs for non- 
malignant (11 cases) and histologically malignant meningiomas ( 7 cases) were $0.45 \%$ and $3.9 \%$, respectively $(p<0.05)$. Nine of 20 meningiomas had a LI greater than $1 \%$, and 6 $(67 \%)$ of those 9 were recurrent. They concluded that meningiomas with a LI greater than $1 \%$ appeared to grow faster and recurred more frequently than those with LIs less than $1 \%$. They suggested that the higher LI might indicate biological malignancy.

Shibuya et al. reported that 53 of 178 meningiomas had BrdU LIs greater than or equal to $1 \%$ [51]. Of these 53 tumors, 21 were diagnosed histopathologically as malignant meningioma. The mean BrdU LI of recurrent tumors (mean \pm SD: $3.9 \pm 2.6 \%$ ) was significantly higher than that of the non-recurrent tumors $(1.9 \pm 1.0 \%) \quad(\mathrm{p}<0.005)$. They showed, by logarithmic regression analysis, that BrdU LI could predict the time to re-operation.

$$
\begin{aligned}
\mathrm{Y}=70.0 \times & \mathrm{X}-1.2(\mathrm{R}=0.76) \\
\mathrm{X} & =\text { BrdU LI }(\%) \\
\mathrm{Y} & =\text { Time interval to re-operation (months) }
\end{aligned}
$$

This formula can be used to predict the period of the recurrence of meningiomas with a high proliferative potential represented by BrdU LI.

Kakinuma et al. [22] reported that the average of BUdR LI for non-recurrent meningiomas was $0.77 \pm 0.13 \%$ (mean $\pm \mathrm{SD})$, and that recurrent meningiomas had significantly higher LI at the first operation $(3.77 \pm 1.22 \%)$. The recurrent ratio significantly increased with the degree of BUdR LI.

Thus, BrdU LI study is useful for the analyses of proliferative potentials and biological behaviors of meningiomas. However, the BrdU LI study is inappropriate for retrospective studies, since it requires intravenous injection of BrdU prior to surgery or incubation of cultured tumor cells with BrdU. Seen in this light, MIB-1 immunohistochemistry has much wider applicability to histopathological studies of tumors, and thus, in this review article, MIB-1 immunohistochemistry is described more extensively as follows.

\section{Expression of Ki-67 Antigen, MIB-1 Staining Index (SI) and Recurrence}

Immunohistochemical staining of the $\mathrm{Ki}-67$ antigen is capable of detecting all proliferative cells [7, 13, 22, 25, 29, 32-34, 42, 44]. Recently, an anti-Ki-67 monoclonal antibody, MIB-1, has become available for analysis of proliferative potentials of tumors using routinely processed paraffin sections [7]. Karamitopoulou et al. used the MIB-1 immunohistochemical staining method to examine the immunoreactivity of Ki-67 antigen in human central nervous system tumors including meningiomas, and found high Ki-67 staining indices even in histologically benign meningiomas [25]. However, this report did not examine the relationship among MIB-1 SI, clinical course, and recurrence.

Many studies have been conducted to examine the relationship between MIB-1 SI and clinical outcome of patients $[1,2,15,18,22,23,26,28-30,32,33,37,40,42,43,46-49$, $54,55]$. Most of the reports showed a significant correlation of MIB-1 SI with clinical outcome [15, 18, 22, 23, 26, 28$30,32,33,42,43,46-48,54,55]$.

\section{1) MIB-1 SI and histopathological subtypes of meningiomas}

In our previous report [32], we examined the proliferative potentials of 127 intracranial meningiomas by MIB-1 immunohistochemical staining method using paraffin sections, and retrospectively analyzed the correlation among MIB-1 SI, histopathological finding, and clinical course of the disease [32]. We found that there was statistical significant difference in the averages of MIB-1 SI between atypical meningioma $(6.1 \%)$ and meningotheliomatous $(2.1 \%)$ or
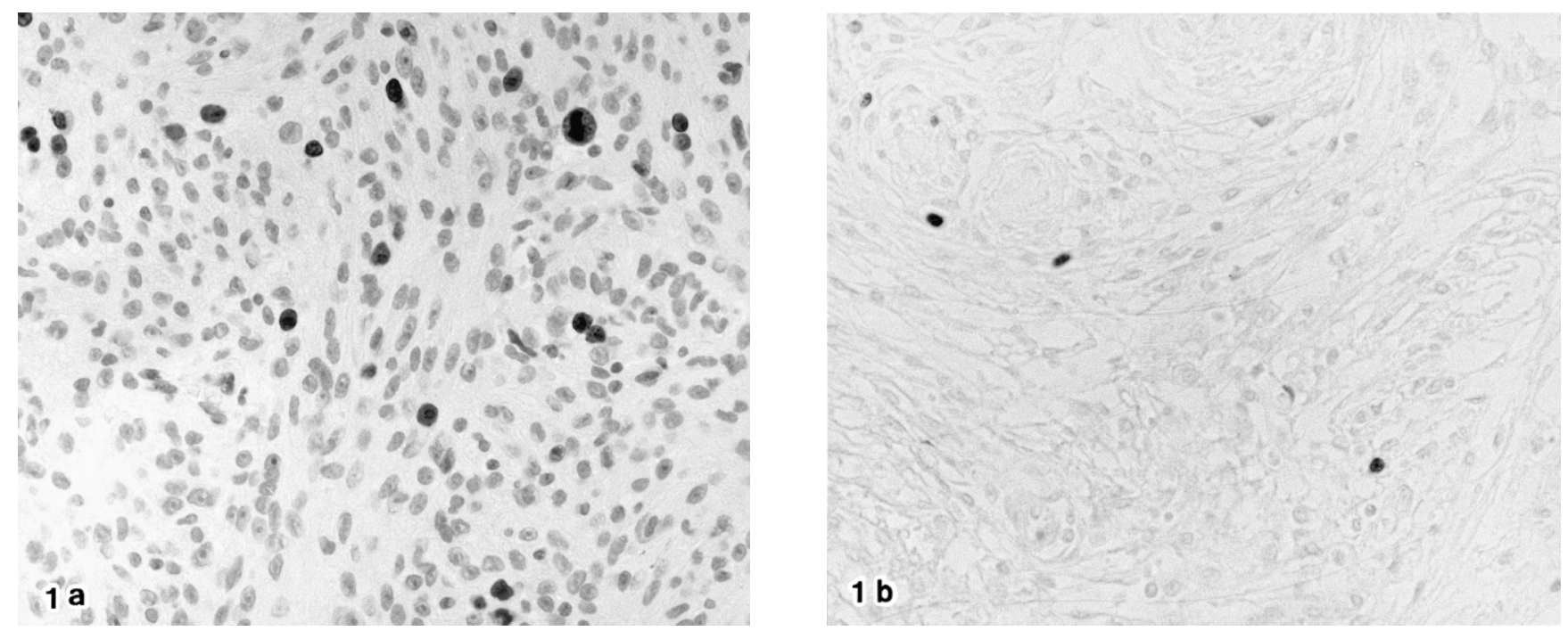

Fig. 1. Representative immunohistochemical stainings of MIB-1. A malignant meningioma with MIB-1 SI of $14.9 \%$ (a: original magnification $\times 200$ ). A meningotheliomatous meningioma with MIB-1 SI of $1.1 \%$ (b: original magnification $\times 200$ ). 
fibroblastic meningioma (3.0\%) [32]. Malignant (anaplastic) meningioma was found to have an extremely high mean MIB-1 SI (26.5\%), compared with that of other subtypes of meningiomas [32]. The representative MIB-1 immunohistochemical stainings are shown in Figure 1. Other several reports have conducted that the mean MIB-1 SI for benign meningiomas ranged from 0.7 to $2.2 \%$, that for atypical meningiomas ranged from 2.1 to $9.3 \%$, and that for anaplastic meningiomas ranged from 11.0 to $16.3 \%[1,26,28,43]$. Perry et al. described that MIB-1 SI appears to be of greatest value in the evaluation of tumors exhibiting borderline atypia [47]. These studies demonstrated that MIB-1 SI is mostly in accordance with the histopathological grading of meningioma.

\section{2) MIB-1 SI and recurrence or regrowth of meningiomas}

We found that mean MIB-1 SIs of non-recurrent tumors, recurrent tumors at the first operation, and recurrent tumors at the re-operation were $1.6 \%, 3.6 \%$, and $8.8 \%$, respectively, and that there were statistically significant differences among these three groups [32] (Fig. 2). Other reports demonstrated that the mean MIB-1 SI of nonrecurrent meningiomas ranged from 0.98 to $3.8 \%$, whereas that of recurrent meningiomas ranged from 4.0 to $8.8 \%$ [1, $28,32,44]$. A recent report by Roser et al. also found that MIB-1 SI is significantly correlated with WHO grading of meningioma [49]. These studies showed a significant correlation of MIB-1 SI with recurrence of meningioma.

\section{3) MIB-1 SI and recurrence or regrowth of meningiomas in relation to the extent of tumor removal}

Some reports have shown that the degree of surgical removal, namely, Simpson's grading, is closely related to future recurrence $[3,8,11,27,29]$. Then, in order to predict the future recurrence of meningioma on the basis of MIB-1 SI of meningiomas more precisely, the tumors should be assigned to each group of Simpson's grade and the relationship between MIB-1 SI and recurrence should be analyzed individually. In our previous report [32], we demonstrated that there is a statistically significant correlation between recurrence and MIB-1 SI in meningiomas that were assigned to each Simpson's grade according to the extent of tumor removal [32]. Roser et al. found that MIB-1 SI was independent of the resection grade, Simpson's grading (grade $1: 5.75 \% ; 2: 4.2 \% ; 3: 6.76 \%$; and 4: 7.99\%) [49]. It may be true that the extent of surgical removal is related to future recurrence; however, in each grading of surgical removal, MIB-1 SI has a relationship with histopathological grading and it is closely related to future recurrence or regrowth of meningiomas.

\section{4) Can we predict the time interval to the next recurrence using MIB-1 SI?}

Time interval to the next recurrence in recurrent meningiomas is associated with proliferative potential as demonstrated by MIB-1 SI, and a formula of correlation is proposed to predict the date of the next recurrence [32] (Fig. 3).

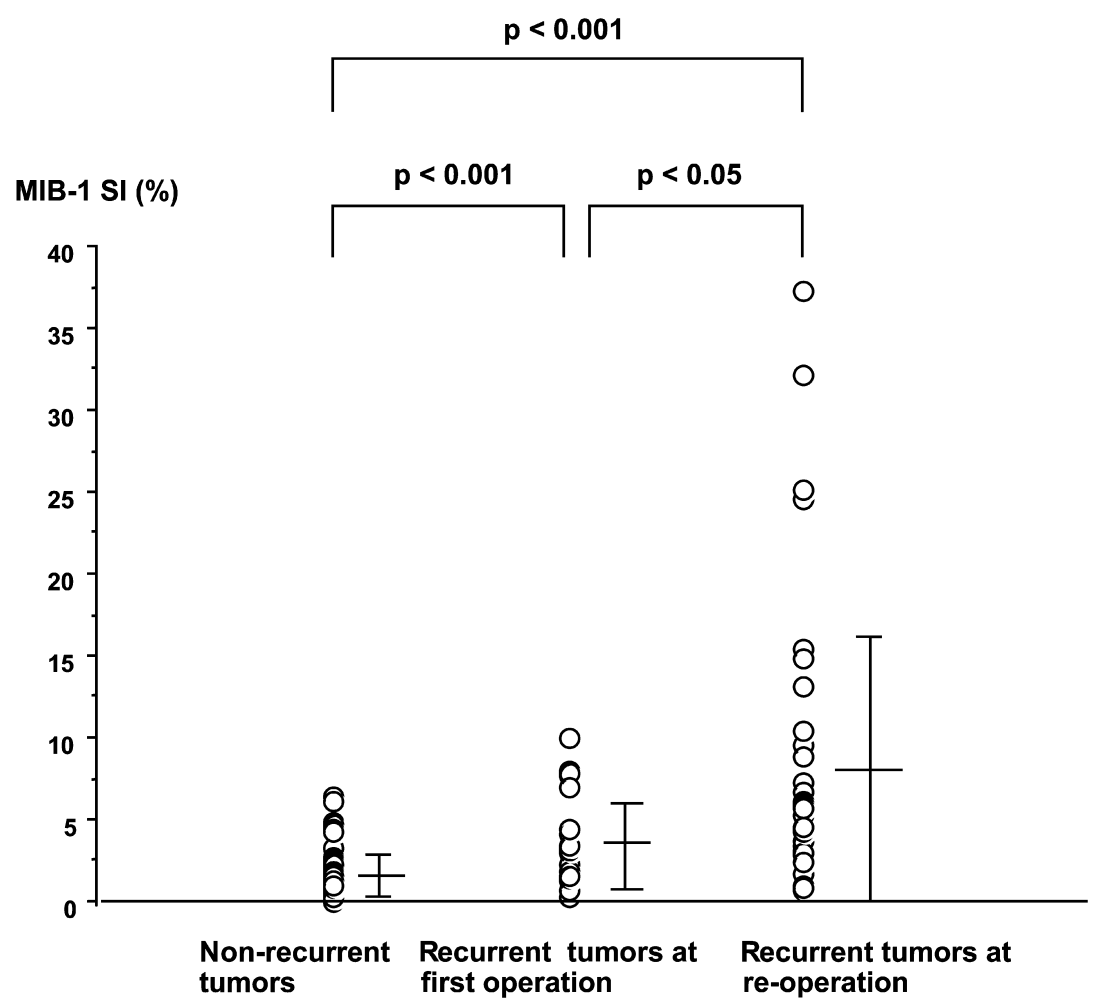

Fig. 2. Comparison of MIB-1 SIs of non-recurrent tumors, recurrent tumors at first operation and recurrent tumors at re-operation (Cited and modified from Ref. 32 with permission). 


$$
\begin{aligned}
\mathrm{Y}=125.82 & -96.447 \times \log (\mathrm{X}) \\
\mathrm{X} & =\text { MIB-1 SI }(\%) \\
\mathrm{Y} & =\text { Time interval to next recurrence (months) }
\end{aligned}
$$

This formula can also be used to predict the period of the recurrence of meningiomas with a high proliferative potential represented by MIB-1 SI.

\section{5) What is the cut-off point of MIB-1 SI that can predict re- currence or regrowth?}

We have shown, by Kaplan-Meier method, that meningiomas with MIB-1 SI of 3\% or higher have significantly high tendency for recurrence in clinical courses, especially in the first 10-year follow-up period [32] (Fig. 4). The other cut-off points predicting recurrence identified in the previous reports were $3.0 \%$ [18], 3.2\% [43], 4.0\% [49], 4.2\% [47], and $10 \%$ [15].

\section{6) Variations of MIB-1 SI among researchers}

We should note that there are some variations among researches of MIB-1 SI of meningiomas. These variations may be due to the differences in staining and counting methodologies among different laboratories. Usually antigen retrieval of $\mathrm{Ki}-67$ is carried out in MIB-1 immunohistochemistry. Munakata and Hendricks stated that microwave oven heating for at least 14 min yielded significant antigen retrieval of Ki-67 in MIB-1 immunohistochemistry using routinely processed paraffin sections [38]. Recent researchers have carried out sufficient antigen retrieval in MIB-1 immunohistochemistry. Therefore, the difference in counting methodology among researchers is the main reason for the variations of MIB-1 SI of meningiomas among researchers. Nakasu et al. evaluated the predictability of MIB-1 immunohistochemistry for growth and recurrences of meningiomas using two different counting methods: (1) in the area of the highest MIB-1 staining (HL method) and (2) in randomly selected fields (RS method). The MIB-1 SI determined by the HL method was approximately twice as high as that by the RS method [43]. Thus, in order to predict the future recurrence or regrowth of meningiomas more precisely, researchers should analyze their own materials and use their standard cut-off points in their own laboratories.

\section{7) Controversies of MIB-1 SI}

There are some reports suggesting that MIB-1 SI is not correlated with tumor behavior [1, 37, 49]. Abramovich and Prayson [1] reported the absence of a significant difference in MIB-1 SI between 32 recurrent and 27 non-recurrent meningiomas. Møller and Brœndstrup [37] also found negative difference in MIB-1 SI between 26 recurrent and 17 non-recurrent meningiomas. They found that PCNA was also of minor value as predictors of recurrence of benign meningiomas. The materials these authors analyzed comprised a relatively small number of tumors. Roser et al. stated that MIB-1 SI was not a statistically significant predictor of survival time in totally excised WHO grade I meningiomas [49]. Their analyses excluded WHO grade 2 and 3 meningiomas and subtotally or partially resected meningiomas. Meanwhile, they proposed that the cut-off point of MIB-1 SI for prediction of future recurrence is $4 \%$. In summary, those reports that found negative correlation between MIB-1 SI and recurrence of meningiomas belong to a minor group. As stated above, most of the reports showed a significant correlation of MIB-1 SI with clinical outcome.

Interval to the next recurrence (months)

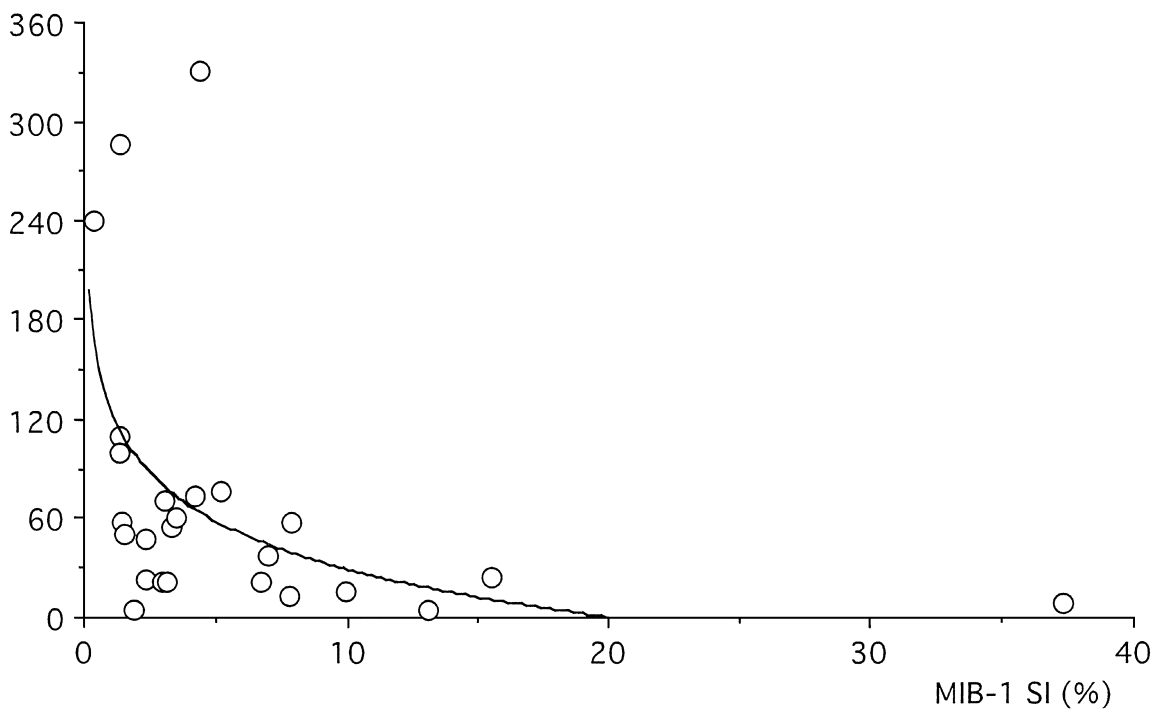

Fig. 3. Correlation between MIB-1 SI and time interval to the next recurrence in patients with recurrent meningioma (Cited and modified from Ref. 32 with permission). 


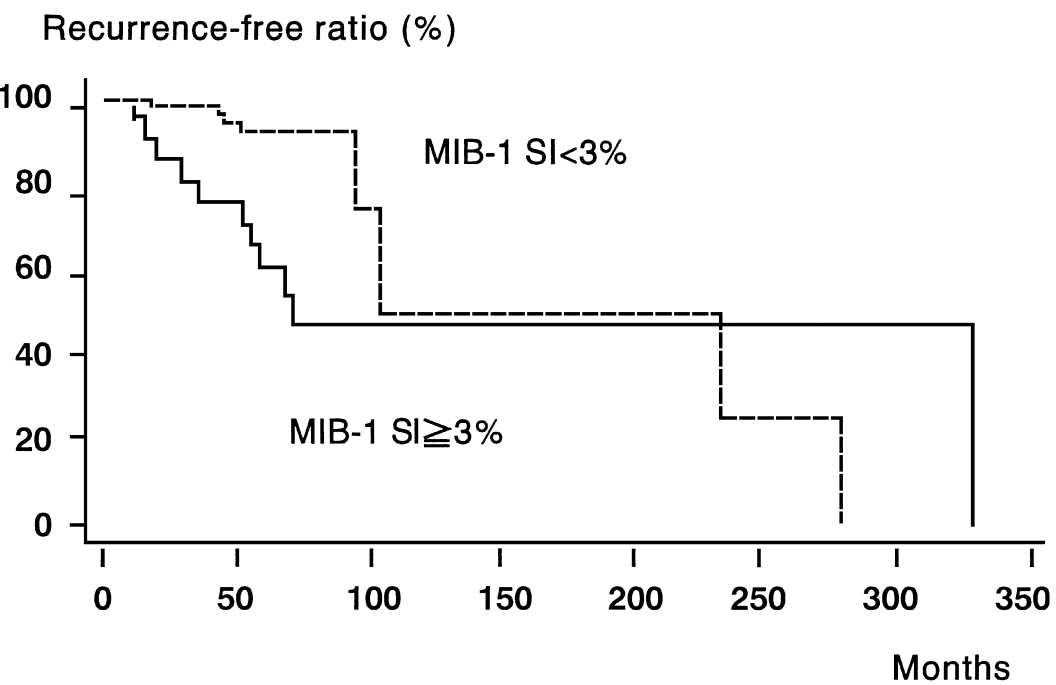

Fig. 4. Kaplan-Meier analysis of recurrence-free ratio and MIB-1 SI (Cited and modified from Ref. 32 with permission).

\section{8) Other factors related to clinical outcome of meningioma and the role of MIB-1 SI in other tumors}

Some authors stated that other factors are also important to predict the future recurrence. Hsu et al. analyzed the expression of transforming growth factor (TGF) $\alpha$ and MIB-1 SI in 57 intracranial meningiomas [18]. They found that the overexpression of TGF $\alpha$ is associated with tumor progression, and that it, as well as MIB-1 SI, is an important prognostic indicator for patients with meningiomas. Yamasaki et al. suggested that high levels of vascular endothelial growth factor (VEGF) expression are significantly related to the recurrence of meningioma, and that VEGF secretion from microscopic residue remaining after surgery may induce neovascularization, which promotes the recurrence of meningioma [55]. As to p53 immunohistochemistry, we found a close relationship among p53 immunoreactivity, MIB-1 SI, and recurrence [33]. Karamitopoulou et al. found that a good correlation exists between histological grading, MIB-1 and p53 protein expression in meningiomas [26]. Nagashima et al. stated that accumulation of p53 protein associated with highly proliferative potentials is a common and characteristic feature that may indicate malignant biological behavior in meningiomas. In contrast, Perry et al. suggested that, regarding patients with primary gross totally resected meningiomas, neither flow cytometry nor p53 immunohistochemistry provides useful prognostic information [47]. Karamitopoulou et al. found that the presence of bcl-2 protein expression, together with high MIB-1 SI, is associated with unfavorable prognosis of the benign meningiomas [26]. Wolfsberger et al. stated that low expression of progesterone receptor is related with the histological grade of meningiomas and MIB-1 SI [54]. Roser et al. found a significant correlation between negative progesterone-receptor status and high tumor vascularity with high MIB-1 SI [49]. The significant correlation of high vascularity and high
MIB-1 SI suggests the neoangiogenetic capacity of meningiomas $[5,19]$. High progesterone receptor status in benign meningiomas is reported to be associated with lower recurrence rate and vice versa $[6,12]$.

MIB-1 SI has been applied to clinical and histopathological studies of other malignant tumors such as gastric cancer $[39,50]$. Setala et al. indicated that p53 protein expression may be of prognostic significance in gastric cancer, while PCNA and Ki-67 antigen expression have no predictive value [50]. Meanwhile, Nagasako et al. stated that the maximum tumor diameter, mitotic index, and Ki-67 antigen expression are useful as an index of malignancy for gastric stromal tumor [39]. Such malignant tumors have more genetic or biological abnormalities than meningiomas, and thus the relevance of MIB-1 SI with clinical outcome may be less important.

To summarize the evaluation of MIB-1 SI in meningiomas in the literature, most reports conducted showed a significant relationship between MIB-1 SI and clinical outcome, although other biological or genetic variables may affect the proliferation of tumors $[15,18,22,23,26,28-30$, $32,33,42,43,46-48,54,55]$. Therefore, the examination of the proliferative potentials of meningiomas using MIB-1 SI is very important for biological and histopathological analyses and the prediction of future recurrence.

\section{Tumor Volume Doubling Time (Td) and Ki-67}

Nakaguchi et al. [42] clarified that MIB-1 immunohistochemical study is useful for determining the rate of regrowth for postoperative residual tumors. They measured tumor volume of 29 intracranial meningiomas from 22 patients by using digital planimeter and computed tomography or magnetic resonance imaging neuroimages. Td was determined by the following formula. 


$$
\begin{aligned}
& \mathrm{Td}=\mathrm{t} \times \log 2 /(\log \mathrm{Vb}-\log \mathrm{Va}) \\
& \text { Va: initial tumor volume } \\
& \text { Vb: tumor volume at } \mathrm{t} \text { days after Va }
\end{aligned}
$$

There was a strong inverse correlation between $\log (\mathrm{Td})$ and MIB-1 SI $(\mathrm{p}<0.001)$.

$$
\log \mathrm{Td}=31.4-0.14 \times \text { MIB-1 SI }
$$

Based on this formula, we can predict the regrowth potential of a meningioma after initial surgery.

\section{BrdU LI and MIB-1 SI}

Kakinuma et al. examined the reciprocal relationship among BrdU LI, MIB-1 SI, and Td of recurrent meningiomas [22]. In their series of 182 primary intracranial meningiomas, 46 cases recurred. The averages of BrdU LI and MIB-1 SI for non-recurrent meningiomas were $0.77 \pm 0.13 \%$ (mean $\pm \mathrm{SD})$ and $4.71 \pm 1.96 \%$, respectively. Meanwhile, the averages of BrdU LI and MIB-1 SI for recurrent meningiomas at the first operation were $3.77 \pm$ $1.22 \%$ and $14.78 \pm 3.17 \%$, respectively. Their linear regression analysis demonstrated a significant correlation between BrdU LI and MIB-1 SI.

$$
\operatorname{BrdU} \mathrm{LI}=0.234+0.98 \times \mathrm{MIB}-1 \mathrm{SI}
$$

They also made accurate calculations by NIH computer software, $\mathrm{Td}$, which showed a significant inverse correlation with each of the indices.

$$
\begin{aligned}
& \mathrm{Td}=-409.609 \times \log \mathrm{BrdU} \mathrm{LI}+368.258 \\
& \mathrm{Td}=-416.008 \times \log \text { MIB- } 1 \mathrm{SI}+730.704
\end{aligned}
$$

They concluded that BrdU LI, MIB-1 SI and Td of individual tumors were well correlated mutually.

Langford et al. [29] analyzed proliferative potentials of meningiomas from 40 adult patients immunohistochemically using BrdU LI and MIB-1 SI. They also found a good correlation between the BrdU and MIB-1 proliferation markers.

Thus, BrdU LI, MIB-1 SI and Td of intracranial meningiomas are considered to have mutual correlation.

\section{Conclusion}

Both BrdU and MIB-1 immunohistochemistry are useful for proliferative potentials of intracranial meningiomas. Many other biological or genetic variables may affect the proliferation of tumors, and nevertheless, BrdU LI and MIB$1 \mathrm{SI}$ are closely related with clinical recurrence or regrowth of meningiomas.

\section{References}

1. Abramovich, C. M. and Prayson, R. A. (1998) MIB-1 labeling indices in benign, aggressive, and malignant meningiomas: a study of 90 tumors. Hum. Pathol. 29; 1420-1427.

2. Abramovich, C. M. and Prayson, R. A. (1999) Histopathologic features and MIB-1 labeling indices in recurrent and nonrecurrent meningiomas. Arch. Pathol. Lab. Med. 123; 793-800.

3. Adegbite, A. B., Khan, M. I., Paine, K. W. E. and Tan, L. K. (1983) The recurrence of intracranial meningiomas after surgical treatment. J. Neurosurg. 58; 51-56.

4. Bagni, A., Botticelli, A., Martinelli, A., Azzoni, P. and Trentini, G. P. (1991) AgNOR counts in recurrent and non-recurrent meningiomas. Histopathology 19; 465-467.

5. Bitzer, M., Opitz, H., Popp, J., Morgalla, M., Gruber, A., Heiss, E. and Voigt, K. (1998) Angiogenesis and brain oedema in intracranial meningiomas: influence of vascular endothelial growth factor. Acta Neurochir. (Wien) 140; 333-340.

6. Brandis, A., Mirzai, S., Tatagiba, M., Walter, G. F., Samii, M. and Ostertag, H. (1993) Immunohistochemical detection of female sex hormone receptors in meningiomas: correlation with clinical and histological features. Neurosurgery 33; 212-217.

7. Cattoretti, G., Becker, M. H. G., Key, G., Duchrow, M., Schlter, C., Galle, J. and Gerdes, J. (1992) Monoclonal antibodies against recombinant parts of the Ki-67 antigen (MIB1 and MIB3) detect proliferating cells in microwave-processed formalin-fixed paraffin sections. J. Pathol. 168; 357-363.

8. Chan, R. C. and Thompson, G. B. (1984) Morbidity, mortality and quality of life following surgery for intracranial meningiomas. J. Neurosurg. 60; 52-60.

9. Chin, L. S. and Hinton, D. R. (1991) The standardized assessment of argyrophilic nucleolar organizer regions in meningiomas. $J$. Neurosurg. 74; 590-596.

10. Christensen, D., Laursen, H. and Klinken, L. (1983) Prediction of recurrence of meningiomas after surgical treatment: A quantitative approach. Acta Neuropathol. 61; 130-134.

11. Crompton, M. R. and Gautier-Smith, P. C. (1970) The prediction of recurrence in meningiomas. J. Neurol. Neurosurg. Psychiatry $33 ; 80-87$.

12. Fewings, P. E., Battersby, R. D. and Timperley, W. R. (2000) Long-term follow up of progesterone receptor status in benign meningioma: a prognostic indicator of recurrence? J. Neurosurg. 92; 401-405.

13. Gerdes, J., Lemke, H., Baisch, H., Wacker, H. H., Schwab, U. and Stein, H. (1984) Cell cycle analysis of a cell proliferationassociated human nuclear antigen defined by the monoclonal antibody Ki-67. J. Immunol. 133; 1710-1715.

14. Gupta, P. K., Sastry Kolluri, V. R., Das, S., Chandra Mouli, B. A., Narayana Swamy, K. S. and Dan, B. S. (1989) Recurrences in meningiomas after surgery. Acta Neurochir. (Wien) 100; 104107.

15. Ho, D. M., Hsu, C. Y., Ting, L. T. and Chiang, H. (2002) Histopathology and MIB-1 labeling index predicted recurrence of meningiomas: a proposal of diagnostic criteria for patients with atypical meningioma. Cancer $94 ; 1538-1547$.

16. Hoshino, T., Nagashima, T., Murovic, J. A., Wilson, C. B. and Davis, R. L. (1986) Proliferative potential of human meningiomas of brain. Cancer $58 ; 1466-1472$.

17. Hoshino, T., Nagashima, T., Cho, K. G., Davis, R. L., Donegan, J., Slusarz, M. and Wilson, C. B. (1989) Variability in the proliferative potential of human gliomas. J. Neurooncol. 7; 137-143.

18. Hsu, D. W., Efird, J. T. and Hedley-Whyte, E. T. (1998) MIB-1 (Ki-67) index and transforming growth factor-alpha (TGF alpha) immunoreactivity are significant prognostic predictors for meningiomas. Neuropathol. Appl. Neurobiol. 24; 441-452.

19. Izycka-Swieszewska, E., Rzepko, R., Borowska-Lehman, J., Baranowska, E. and Warzocha, D. (1999) Recurrent meningiomas-the immunohistochemical analysis of angiogenesis and cellular proliferation. Preliminary study. Folia Neuropathol. 37; $179-184$

20. Jaaskelainen, J., Haltia, M. and Servo, A. (1986) Atypical and 
anaplastic meningiomas: radiology, surgery, radiotherapy, and outcome. Surg. Neurol. 25; 233-242.

21. Jellinger, K. and Slowik, F. (1975) Histological subtypes and prognostic problems in meningiomas. J. Neurol. 208; 279-298.

22. Kakinuma, K., Tanaka, R., Onda, K. and Takahashi, H. (1998) Proliferative potential of recurrent intracranial meningiomas as evaluated by labelling indices of BUdR and $\mathrm{Ki}-67$, and tumour doubling time. Acta Neurochir. (Wien) 140; 26-31, discussion 31-32.

23. Kalala, J. P., Benoit, D. and De Ridder, L. (2004) Can recurrence of meningiomas be predicted? Anticancer Res. 24; 2319-2324.

24. Karamitopoulou, E., Perentes, E., Melachrinou, M. and Maraziotis, T. (1993) Proliferating cell nuclear antigen immunoreactivity in human central nervous system neoplasms. Acta Neuropathol. 85; 316-322.

25. Karamitopoulou, E., Perentes, E., Diamantis, I. and Maraziotis, T. (1994) Ki-67 immunoreactivity in human central nervous system neoplasms: a study with MIB1 monoclonal antibody on archival material. Acta Neuropathol. 87; 47-54.

26. Karamitopoulou, E., Perentes, E., Tolnay, M. and Probst, A. (1998) Prognostic significance of MIB-1, p53, and bcl-2 immunoreactivity in meningiomas. Hum. Pathol. 29; 140-145.

27. Kleihues, P. and Cavenee, W. K. (2000) WHO classification of tumours of the nervous system. In "Pathology and Genetics of Tumours of the Nervous System", ed. by P. Kleihues and W. K. Cavenee, IARC Press, International Agency for Research on Cancer, Lyon, pp. 6-7.

28. Kolles, H., Niedermayer, I., Schmitt, C., Henn, W., Feld, R., Steudel, W. I., Zang, K. D. and Feiden, W. (1995) Triple approach for diagnosis and grading of meningiomas: histology, morphometry of Ki-67/Feulgen stainings, and cytogenetics. Acta Neurochir. (Wien) 137; 174-181.

29. Langford, L. A., Cooksley, C. S. and DeMonte, F. (1996) Comparison of MIB-1 (Ki-67) antigen and bromodeoxyuridine proliferation indices in meningiomas. Hum. Pathol. 27; 350-354.

30. Madsen, C. and Schroder, H. D. (1997) Ki-67 immunoreactivity in meningiomas-determination of the proliferative potential of meningiomas using the monoclonal antibody Ki-67. Clin. Neuropathol. 16; 137-142.

31. Marks, S. M., Whitwell, H. L. and Lye, R. H. (1986) Recurrence of meningiomas after operation. Surg. Neurol. 25; 436-440.

32. Matsuno, A., Fujimaki, T., Sasaki, T., Nagashima, T., Ide, T., Asai, A., Matsuura, R., Utsunomiya, H. and Kirino, T. (1996) Clinical and histopathological analysis of proliferative potentials of recurrent and non-recurrent meningiomas. Acta Neuropathol. 91; 504-510.

33. Matsuno, A., Nagashima, T., Matsuura, R., Tanaka, H., Hirakawa, M., Murakami, M., Tamura, A. and Kirino, T. (1996) Correlation between MIB-1 staining index and the immunoreactivity of p53 protein in recurrent and non-recurrent meningiomas. Am. J. Clin. Pathol. 106; 776-781.

34. Matsuno, A., Sasaki, T., Nagashima, T., Kirino, T. and Osamura, R. Y. (2001) Proliferative potentials of intracranial chordomas with a reference to extracranial chordoma. Acta Histochem. Cytochem. 34; 305-312.

35. Melamed, S., Sahar, A. and Beller, A. J. (1979) The recurrence of intracranial meningioma. Neurochirurgia $22 ; 47-51$.

36. Mirimanoff, R. O., Dosoretz, D. E., Linggood, R. M., Ojemann, R. G. and Martuza, R. L. (1985) Meningioma: analysis of recurrence and progression following neurosurgical resection. $J$. Neurosurg. 62; 18-24.

37. Møller, M. L. and Brœndstrup, O. (1997) No prediction of recurrence of meningiomas by PCNA and Ki-67 immunohistochemistry. J. Neurooncol. 34; 241-246.

38. Munakata, S. and Hendricks, J. B. (1993) Effect of fixation time and microwave oven heating time on retrieval of the Ki-67 anti- gen from paraffin-embedded tissue. J. Histochem. Cytochem. 41; 1241-1246.

39. Nagasako, Y., Misawa, K., Kohashi, S., Hasegawa, K., Okawa, Y., Sano, H., Takada, A. and Sato, H. (2003) Evaluation of malignancy using Ki-67 labeling index for gastric stromal tumor. Gastric Cancer 6; 168-172.

40. Nagashima, G., Aoyagi, M., Yamamoto, M., Yamamoto, S., Wakimoto, H., Ohno, K., Yamamoto, K. and Hirakawa, K. (1999) P53 overexpression and proliferative potential in malignant meningiomas. Acta Neurochir. (Wien) 141; 53-61.

41. Nagashima, T., Hoshino, T., Cho, K. G., Edwards, M. S., Hudgins, R. J. and Davis, R. L. (1988) The proliferative potential of human ependymomas measured by in situ bromodeoxyuridine labeling. Cancer 61; 2433-2438.

42. Nakaguchi, H., Fujimaki, T., Matsuno, A., Matsuura, R., Asai, A., Suzuki, I., Sasaki, T. and Kirino, T. (1999) Postoperative residual tumor growth of meningioma can be predicted by MIB-1 immunohistochemistry. Cancer 85; 2249-2254.

43. Nakasu, S., Li, D. H., Okabe, H., Nakajima, M. and Matsuda, M. (2001) Significance of MIB-1 staining indices in meningiomas: comparison of two counting methods. Am. J. Surg. Pathol. 25; 472-478.

44. Ohta, M., Iwaki, T., Kitamoto, T., Takeshita, I., Tateishi, J. and Fukui, M. (1994) MIB1 staining index and scoring of histologic features in meningiomas: Indicators for the prediction of biologic potential and proliferative management. Cancer 74; 3176-3189.

45. Orita, T., Kajiwara, K., Nishizaki, T., Ikeda, N., Kamiryo, T. and Aoki, H. (1990) Nucleolar organizer regions in meningioma. Neurosurgery 26; 43-46.

46. Paulus, W., Meixensberger, J., Hofmann, E. and Roggendorf, W. (1993) Effect of embolisation of meningioma on Ki-67 proliferation index. J. Clin. Pathol. 46; 876-877.

47. Perry, A., Stafford, S. L., Scheithauer, B. W., Suman, V. J. and Lohse, C. M. (1998) The prognostic significance of MIB-1, p53, and DNA flow cytometry in completely resected primary meningiomas. Cancer 82; 2262-2269.

48. Prayson, R. A. (1996) Malignant meningioma: a clinicopathologic study of 23 patients including MIB1 and p53 immunohistochemistry. Am. J. Clin. Pathol. 105; 719-726.

49. Roser, F., Samii, M., Ostertag, H. and Bellinzona, M. (2004) The $\mathrm{Ki}-67$ proliferation antigen in meningiomas. Experience in 600 cases. Acta Neurochir. (Wien) 146; 37-44.

50. Setala, L., Kosma, V. M., Lipponen, P., Naukkarinen, A., Nordling, S., Hollmen, S., Eskelinen, M., Syrjanen, K. and Alhava, E. (1998) Clinical relevance of p53 index and expression of proliferating cell nuclear antigen and $\mathrm{Ki}-67$ in gastric cancer. J. Cancer Res. Clin. Oncol. 124; 497-502.

51. Shibuya, M., Hoshino, T., Ito, S., Wacker, M. R., Prados, M. D., Davis, R. L. and Wilson, C. B. (1992) Meningiomas: clinical implications of a high proliferative potential determined by bromodeoxyuridine labeling. Neurosurgery 30; 494-498.

52. Simpson, D. (1957) The recurrence of intracranial meningiomas after surgical treatment. J. Neurol. Neurosurg. Psychiatry 20;2239.

53. Tsutsumi, Y. and Fushiki, S. (2000) Comparison of cell kinetics between the boundary and the interboundary areas during hindbrain segmentation in the chick embryo. Acta Histochem. Cytochem. 33; 141-147.

54. Wolfsberger, S., Doostkam, S., Boecher-Schwarz, H. G., Roessler, K., van Trotsenburg, M., Hainfellner, J. A. and Knosp, E. (2004) Progesterone-receptor index in meningiomas: correlation with clinico-pathological parameters and review of the literature. Neurosurg. Rev. 27; 238-245.

55. Yamasaki, F., Yoshioka, H., Hama, S., Sugiyama, K., Arita, K. and Kurisu, K. (2000) Recurrence of meningiomas. Cancer 89; $1102-1110$. 